\title{
MODELO PARA LA EVALUACIÓN DE HABILIDADES EN INGENIERÍA AUTOMÁTICA
}

\section{MODEL FOR ASSESSMENT OF SKILLS IN AUTOMATION ENGINEERING}

Omar Mar Cornelio ${ }^{1}$ Jorge González Gulín²

2. Máster en Informática Aplicada, Profesor Auxiliar de la Facultad Ciencias y Tecnologías Computacionales, Universidad de las Ciencias Informática. Cuba. E-mail: omarmar@uci.cu

3. Licenciado en Física Pura, Doctor en Ciencias Físicas, Profesor Titular, Director del Centro de Estudio de la Matemática Computacional, Universidad de las Ciencias Informática. Cuba. E-mail: gulin@uci.cu

\section{Citación sugerida:}

Mar Cornelio, O. y González Gulín, J. (2018). Modelo para la evaluación de habilidades en Ingeniería Automática. 3C TIC: Cuadernos de desarrollo aplicados a las TIC, 7(1), 21-32. DOI: <http://dx.doi.org/10.17993/3ctic.2018.59.21-32/>. 
Con la introducción de los Sistemas de Laboratorios a Distancias en la enseñanza de la Automática, se crea una nueva forma de materializar los objetivos y contenido del Proceso Docente Educativo. En este sentido, la evaluación constituye un elemento importante para comprobar el aprendizaje de los estudiantes. En la Carrera de Automática de la Universidad Central "Martha Abreu" de Las Villas se realiza a partir de la valoración realizada sobre el resultado de las prácticas de laboratorios. Sin embargo, es posible que personas no competentes accedan a las prácticas preparadas causando un mal funcionamiento en los dispositivos acoplado. La presente investigación describe una solución a la problemática planteada a partir de la elaboración de un modelo para la evaluación de competencias implementado sobre una herramienta informática, se realiza una valoración sobre la aplicabilidad de la propuesta a partir de la medición de la satisfacción de usuario con la técnica ladov donde se obtiene un alto índice de satisfacción.

\section{ABSTRACT}

With the introduction of Laboratory Systems to Distances in the teaching of Automatic, a new way of materializing the objectives and content of the Educational Teaching Process is created. In this sense, evaluation is an important element to verify student learning. In the Automatic Course of the Central University "Martha Abreu" of Las Villas is made from the assessment made on the results of laboratory practices. However, it is possible for noncompetent persons to access the prepared practices causing a malfunction in the coupled devices. The present research describes a solution to the problematic raised from the elaboration of a model for the evaluation of competences implemented on a computer tool. An assessment is made on the applicability of the proposal based on the measurement of user satisfaction with the ladov technique where a high satisfaction index is obtained.

\section{PALABRAS CLAVE}

Evaluación de competencias, Sistema de laboratorios a distancia, Educación a distancia, Modelo.

\section{KEYWORDS}

Evaluation of competences, System of distance laboratories, Distance education, Model. 


\section{INTRODUCCIÓN}

Con el desarrollo de las Tecnologías de la Información y las Comunicaciones (TIC) y el uso de Internet, se extiende su dominio de aplicación a todas las áreas de conocimiento. La Educación implementa sus novedosas tecnologías educativas como nueva forma de materializar los objetivos y contenidos del Proceso Docente Educativo (Sáez, 2012). Partiendo de la nueva forma de interacción, surge la Educación a Distancia (Heedy \& Uribe, 2008) donde el alumnado materializa los conocimientos adquiridos en laboratorios equipados en correspondencia con la asignatura o perfil que se estudie o de manera virtual mediante las simulaciones de la realidad (González \& Cabero, 2009).

La enseñanza de la ingeniería se beneficia con la implementación de sus múltiples plataformas educativas, donde los Sistemas de Laboratorios a Distancias permiten que estudiantes realicen prácticas de laboratorios reales a través del uso de Internet (Samuelsen \& Graven, 2016). En la Universidad Central "Martha Abreu" de las Villas, para la carrera de Automática se implementa una plataforma a distancia donde los estudiantes pueden diseñar controladores digitales para manipular dispositivos físicos (Santana, Ferre, Izaguirre, Aracil, \& Hernandez, 2013) contribuyendo así a su formación. Sin embargo, las estaciones acopladas a los Sistemas de Laboratorios a Distancia suponen como dificultad que no es posible saber si la persona que realiza la práctica de laboratorio es competente o está capacitada, generándose en ocasiones afectación de la dinámica de los dispositivos (Samuelsen \& Graven, 2016). En este sentido, la evaluación constituye un elemento importante para comprobar el aprendizaje de los estudiantes (Francedy, 2011).

La presente investigación describe una solución a la problemática planteada mediante la implementación de un modelo para la evaluación de competencias codificado mediante una herramienta informática. Se define como objetivo del presente modelo: diagnosticar las habilidades profesionales de los estudiantes que realizan prácticas de laboratorios contribuyendo al control de acceso y sistema de recomendaciones en el Sistema de Laboratorios a Distancias.

\section{METODOLOGÍA}

El modelo posee estructura, componentes, cualidades y principios encaminados a satisfacer el objetivo definido. La Figura 1 muestra una representación gráfica de los elementos antes mencionados que se integran en el modelo propuesto. 


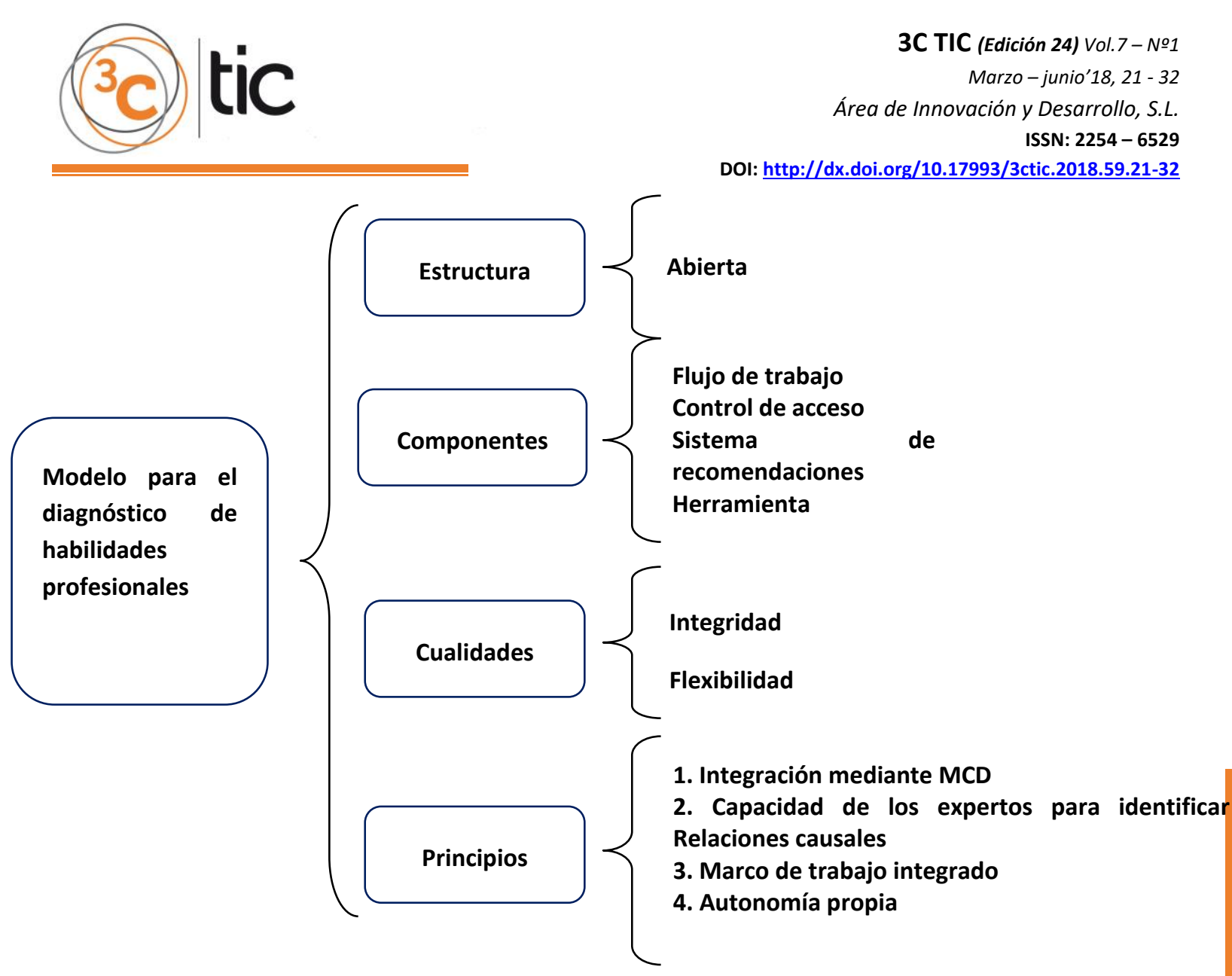

Figura 1. Estructura, componentes, cualidades y principios del modelo.

El modelo posee una estructura abierta y colaborativa evidenciada mediante la generación e intercambio de información. A partir de la información de entradas se conforman los modelos mentales que representan las relaciones causales de los diferentes conceptos que intervienen en el diagnóstico de habilidades. Como resultado, se obtiene una representación del conocimiento expresado por los expertos en forma de MCD (Bello R, González S, Martín A, \& $M, 2015)$.

El modelo se ha diseñado mediante cuatro componentes fundamentales a través de cuyo funcionamiento se garantiza su consistencia. Ellos son:

Flujo de trabajo: el flujo de trabajo está compuesto por en siete actividades fundamentales. A continuación se presenta una breve descripción de cada una de ellas, en posteriores secciones del capítulo se realiza una descripción detallada:

1. Identificar las habilidades profesionales: esta actividad se realiza al inicio del proceso, representa un valor de entrada con el que se nutre el modelo. Las habilidades son obtenidas mediante la consulta a expertos.

2. Obtener y agregar los mapas cognitivos difusos: permite realizar una representación del conocimiento causal del grupo de experto que interviene en el proceso.

3. Realizar el análisis estático: a partir del análisis estático, es posible identificar conocimiento antes oculto atribuido al conjunto de habilidades profesionales identificadas. 
4. Procesar evaluación de los practicantes: permite a partir del desarrollo de los cuestionarios evaluativos se obtienen las preferencias de los usuarios que representa un parámetro de entrada para determinar realizar el diagnóstico de habilidades profesionales.

5. Determinar el control de acceso: a partir del conjunto de preferencia y habilidades profesionales con la utilización de técnicas de inteligencia artificial se determina si una alternativa puede realizar las prácticas de laboratorios en el sistema físico.

6. Generar el sistema de recomendaciones: a partir que se identifique que una alternativa no posee las habilidades requeridas para realizar las prácticas, el sistema de recomendaciones brinda un conjunto de elementos a tener en cuenta para mejorar el desempeño de la alternativa practicante.

7. Realizar análisis de estabilidad: permite una vez concedido el acceso al sistema físico determinar si la estrategia de control diseñada es correcta para lo cual se le realiza un análisis de estabilidad a partir de la ubicación geométrica de las raíces.

Control de acceso: una deficiencia que supone el trabajo en los Sistemas de Laboratorios a Distancias es que personas no competentes puedan acceder a los recursos compartidos mediante las prácticas de laboratorios y se genere un mal funcionamiento de los dispositivos. El componente control de acceso a partir del diagnóstico de las habilidades profesionales determina si una persona puede realizar o no una práctica solicitada.

Sistema de recomendaciones: el componente para la producción automática de recomendaciones permite, a partir del comportamiento de los estudiantes que realizan las prácticas de laboratorios, sugerir cuales son las habilidades que deben potenciar y hacia ellas orientar el desarrollo de sus prácticas.

Herramienta: en el modelo se incluye como componente un Sistema de Laboratorios a Distancia que soporta la ejecución de las actividades del flujo de trabajo. El Sistema de Laboratorios a Distancia permite aprovechar las potencialidades de Internet para garantizar un ambiente multiusuario (Mar, Gulín, \& I, 2016).

Análisis de estabilidad: es el componente del modelo que garantiza la protección final a los dispositivos acoplados en el Sistema de Laboratorios a Distancia, permite evaluar las estrategias de control diseñadas por los estudiantes que realizan prácticas de laboratorios.

Las cualidades que distinguen al modelo son:

- Integración: el modelo garantiza la interconexión de sus componentes en combinación para el diagnóstico de habilidades, control de acceso y sistema de recomendaciones. Se implementan técnicas de Inteligencia Artificial en una herramienta informática para el soporte tecnológico de la solución.

- Flexibilidad: utiliza 2-tuplas para representar la incertidumbre en las relaciones causales que incluyen la obtención de los modelos mentales. Se adapta a un ambiente bajo incertidumbre manteniendo un comportamiento coherente ante cada entrada de información.

- Interdependencia: el modelo toma como punto de partida los datos de entrada obtenidos del modo de actuación de los estudiantes que interactúan con el Sistema 


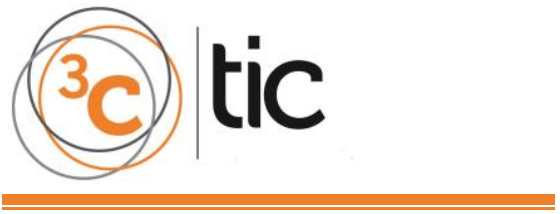

de Laboratorios a Distancia. Los resultados analizados contribuyen a una base de experiencia que conforma el registro del estudiante que realiza las prácticas de laboratorios.

Sustenta los siguientes principios:

- Propone la integración mediante mapas cognitivos difuso en el diagnóstico de habilidades profesionales.

- Supone la capacidad del equipo de expertos para la correcta identificación de las relaciones causales.

- Orientado hacia la identificación a partir de las relaciones causales de las necesidades de capacitación.

- Define un marco de trabajo único para la integración de los componentes del modelo.

- Supone autonomía propia para el diagnóstico de habilidades.

\subsection{ESTRUCTURA GENERAL DEL MODELO PARA EL DIAGNÓSTICO DE HABILIDADES PROFESIONALES}

El modelo está orientado a soportar el flujo de trabajo para el diagnóstico de habilidades. Realiza cuatro actividades básicas: entrada, almacenamiento, procesamiento y salida de información como se describe a continuación (Mar, I, \& Gulín, 2017):

1. Entrada de información

Parte del proceso mediante el cual el modelo toma los datos que requiere para almacenar y procesar las informaciones necesarias. Las entradas pueden ser manuales o automáticas. Las manuales son aquellas que se proporcionan de forma directa por un usuario como las habilidades profesionales, la gestión de las relaciones causales introducidas por los expertos, las respuestas a los cuestionarios elaborados para diagnosticar las habilidades, etc. Las automáticas son datos o información que provienen o son tomados de otras actividades o componentes como los pesos atribuidos a una habilidad.

\section{Almacenamiento de información}

El almacenamiento es la actividad o capacidad más importante que garantiza el modelo, ya que a través de esta propiedad se puede recordar la información guardada en la actividad anterior. Para el modelo propuesto, los datos derivados del proceso de entrada de información son almacenados para su posterior uso mediante la actividad procesamiento de la información.

\section{Procesamiento de información}

Capacidad del modelo para efectuar cálculos de acuerdo con una secuencia de operaciones preestablecida. Esta característica permite la transformación de datos en información organizada. Interviene directamente en este proceso, siendo posible determinar el umbral de las habilidades profesionales de una persona, inferir si la persona está capacitada para 
realizar una práctica de laboratorios o determinar las necesidades de capacitación de una persona.

4. Salida de información

La salida es la capacidad del modelo para retroalimentar a las personas que intervienen en el proceso. Para el modelo propuesto, existe un componente destinado a la herramienta informática donde es posible visualizar las informaciones de salida que son necesarias transmitir a los usuarios.

La figura 2 Presenta un esquema general de las actividades que conforman el modelo.

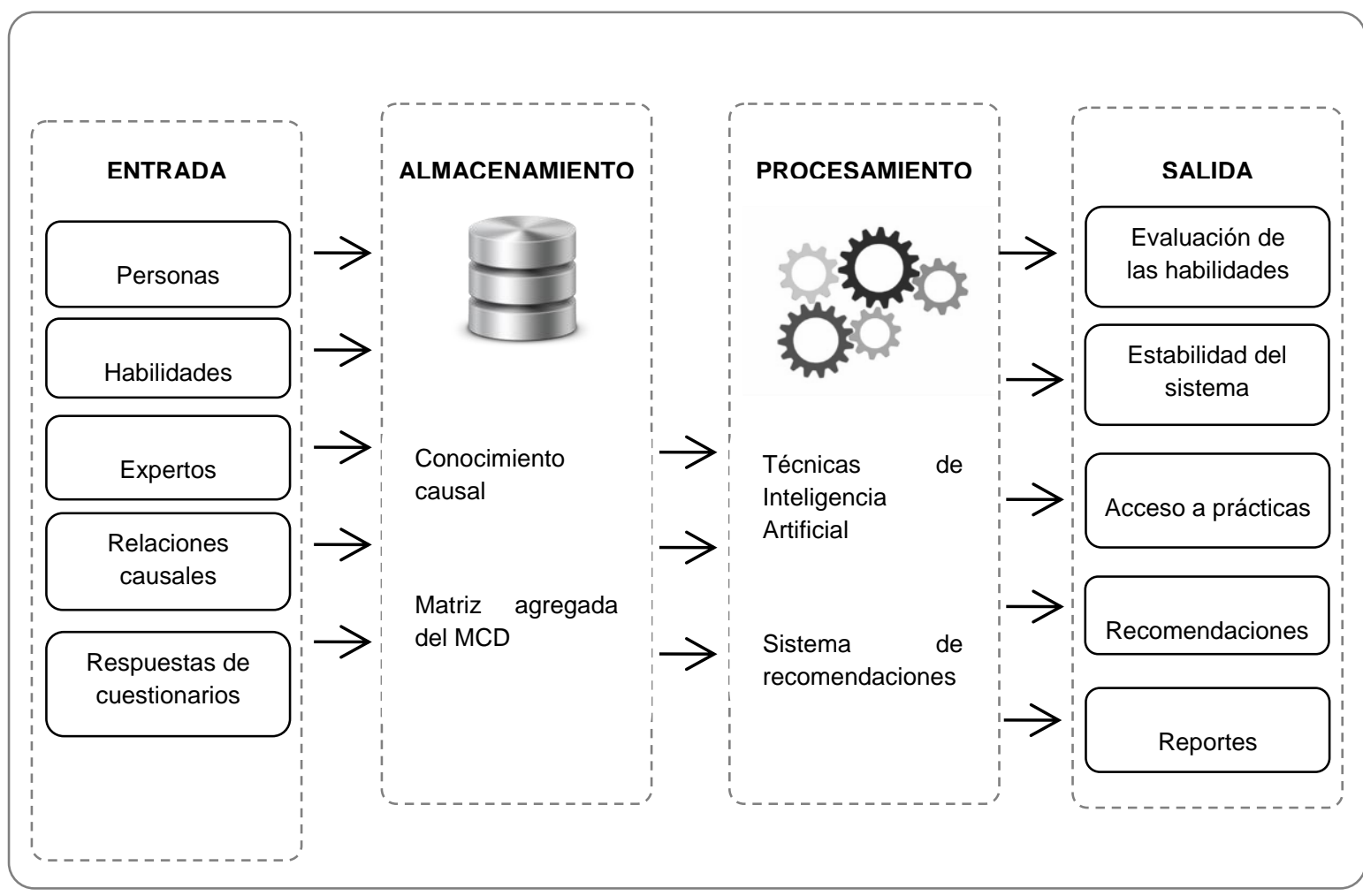

Figura 2. Esquema general de las actividades del modelo.

\section{RESULTADOS Y DISCUSIÓN}

En investigaciones donde el resultado final impacta sobre un conjunto de personas, la valoración para medir la satisfacción del usuario final representa una vía para validar empíricamente la propuesta presentada. La técnica ladov constituye una forma de medir el grado de satisfacción creada por Kuzmina (Kuzmina, 1970).

La técnica se basa en la aplicación de una encuesta compuesta por preguntas cerradas y abiertas (Castillo \& Ginoris, 2005). Las tres preguntas cerradas establecen una relación en el Cuadro Lógico de ladov (López \& González, 2002), indicando la escala de satisfacción individual de cada encuestado, mientras que las preguntas abiertas permiten profundizar en los elementos positivos y las recomendaciones o insuficiencias de la propuesta que se evalúa. 


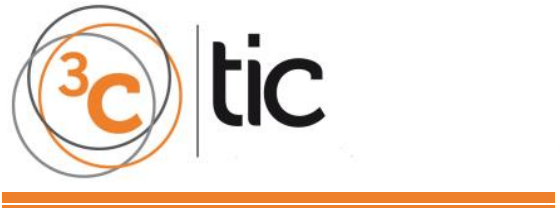

La Tabla 1 presenta el conjunto de etiquetas lingüísticas a las que le pertenecen los valores numéricos utilizados para medir la satisfacción individual de cada encuestado.

Tabla 1. Conjunto de términos empleado como escala valorativa.

\begin{tabular}{|l|l|}
\hline Etiquetas lingüísticas que se representa & Valor \\
\hline Clara satisfacción & 1 \\
\hline Más satisfecho que insatisfecho & 2 \\
\hline No definida & 3 \\
\hline Más insatisfecho que satisfecho & 4 \\
\hline Clara insatisfacción & 5 \\
\hline Contradictoria & 6 \\
\hline
\end{tabular}

Para la implementación de la técnica se aplicó un cuestionario a un grupo de profesores y directivos después de interactuar con el modelo propuesto. El objetivo del instrumento realizado estuvo intencionado en función de validar la satisfacción de los usuarios finales.

La muestra utilizada para el desarrollo de la actividad estuvo compuesta por cinco profesores de la Universidad Central " Martha Abreu" de las Villas, cuatro profesores de la Universidad de Oriente, dos profesores del Instituto Superior Politécnico "José Antonio Echeverría", dos profesores de la Universidad de las Ciencias Informáticas y dos profesores de la Universidad de Pinar del Rio. Para un total de 15 usuarios de ellos 3 jefes de Departamentos Docentes representando 5 instituciones nacionales.

Las variables evaluadas fueron las siguientes:

- Satisfacción del cliente: el colectivo de profesores y directivos consideran que el modelo propuesto satisface las necesidades de información para apoyar el diagnóstico de habilidades profesionales y su contribución al control de acceso de las prácticas de laboratorios.

- Aplicabilidad del modelo: el colectivo de profesores y directivos consideran que la concepción del modelo y la herramienta es aplicable en el Sistema Educacional Cubano.

- Utilidad del modelo: el colectivo de profesores y directivos consideran que la aplicación del modelo propuesto garantiza el diagnóstico de habilidades profesionales y contribuye al control de acceso de las prácticas de laboratorios.

Los valores obtenidos como resultados de las preferencias del conjunto de usuarios que intervino en el proceso, son presentados en el Cuadro Lógico de ladov tal como se muestra en la tabla 2. 
Tabla 2. Cuadro Lógico de ladov para la investigación.

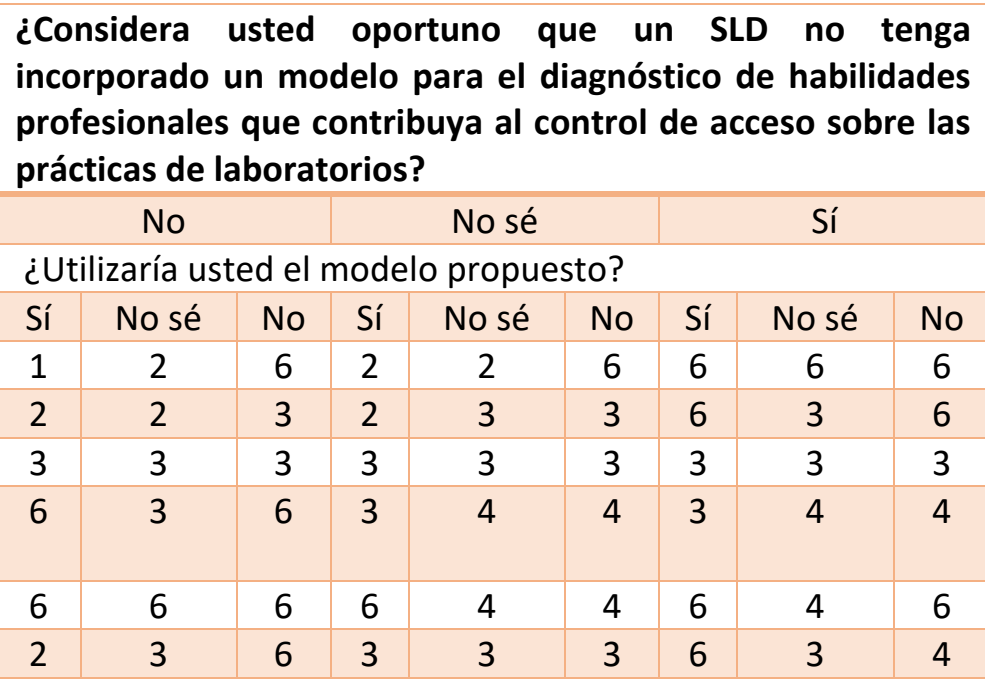

Para obtener el índice de satisfacción grupal (ISG), se trabaja con los diferentes niveles de satisfacción que expresan en la escala numérica 1 y -1 tal como refiere la tabla 3.

Tabla 3. Conjunto de términos empleado como nivel de satisfacción.

\begin{tabular}{|l|c|}
\hline Etiquetas lingüísticas que se representa & Valor \\
\hline Máximo de satisfacción & 1 \\
\hline Más satisfecho que insatisfecho & 0,5 \\
\hline No definido y contradictorio & 0 \\
\hline Más insatisfecho que satisfecho & $-0,5$ \\
\hline Máxima insatisfacción & -1 \\
\hline
\end{tabular}

Una vez aplicadas las encuestas se obtuvieron los resultados en la escala de satisfacción individual tal como presenta la tabla 4:

Tabla 4. Resultado de la satisfacción sobre la aplicación de la técnica ladov.

\begin{tabular}{|l|l|l|}
\hline Nivel de satisfacción & Cantidad & Porciento \\
\hline Máxima satisfacción & 12 & $80 \%$ \\
\hline Más satisfecho que insatisfecho & 2 & $13,33 \%$ \\
\hline No definida o contradictoria & 1 & $6,66 \%$ \\
\hline Más insatisfecho que satisfecho & 0 & 0,00 \\
\hline Máxima insatisfacción & 0 & 0,00 \\
\hline
\end{tabular}

A partir de la aplicación de la técnica, se obtiene como resultado el índice de Satisfacción Grupal (ISG) que representa un parámetro atribuido a la concordancia del grupo de usuarios a los que se les aplicó el instrumento. El ISG se determina mediante la ecuación 1.

$$
I S G=\frac{A(+1)+B(0.5)+C(0)-D(0.5)+E(-1)}{N}
$$




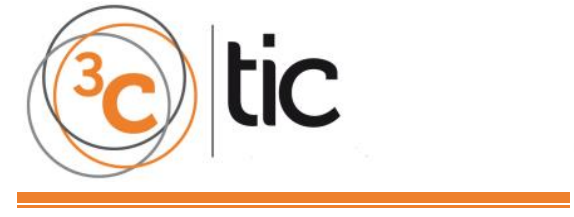

Donde:

DOI: http://dx.doi.org/10.17993/3ctic.2018.59.21-32

A, B, C, D, E: representa el número de sujetos con índice individual 1,2, (3 ० 6), 4,5.

N: representa el número total de usuarios del grupo.

El Índice de Satisfacción Grupal arroja valores comprendidos entre +1 y -1 tal como se muestra en la figura 3. Los valores comprendidos entre -1 y $-0,5$ indican insatisfacción, los valores comprendidos entre -0.49 y 0.49 evidencian contradicciones por lo que se expresa como insatisfacción y los valores comprendidos entre 0,5 y 1 indican que existe satisfacción.

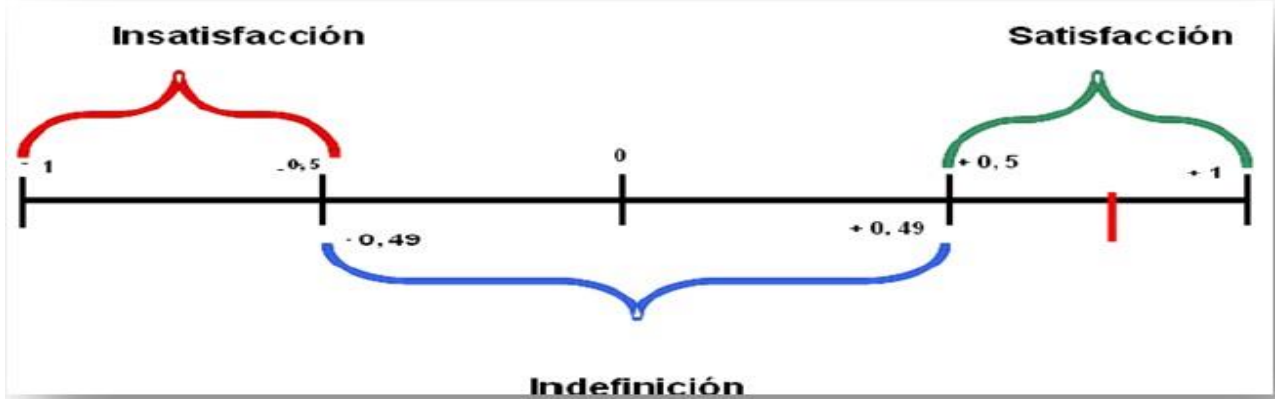

Figura 3. Índice de satisfacción (LÓPEZ \& GONZÁLEZ, 2002).

En el proceso realizado se obtuvo un ISG $=0.866$ considerando que existe alta satisfacción. $A$ partir de obtener un ISG satisfactorio, se realiza el siguiente análisis:

- El criterio de los encuestados respecto a si ¿Considera usted oportuno que un SLD no tenga incorporado un modelo para el diagnóstico de habilidades profesionales que contribuya al control de acceso sobre las prácticas de laboratorios? obtuvo una máxima satisfacción de un $86,66 \%$, un 13,33 \% lo consideró más satisfecho que insatisfecho.

- Respecto a ¿Cuál es su criterio sobre el Modelo para la evaluación de habilidades profesionales? el $86,6 \%$ se considera con máxima satisfacción y el otro $13,33 \%$ lo calificó más satisfecho que insatisfecho.

- En cuanto a si ¿Utilizaría usted el modelo propuesto?, el 80 \% se considera con la máxima satisfacción, el 13,33 \% se califica más satisfecho que insatisfecho y el otro $6,66 \%$ se califica como no definida o contradictoria.

Además de la satisfacción obtenida, se pudo identificar mediante las preguntas abiertas las siguientes fortalezas:

- A partir del funcionamiento general del modelo, sería oportuno extender a otras áreas del conocimiento su dominio de aplicación.

- Que se extienda el modelo para medir habilidades profesionales no solo en los Sistemas de Laboratorios a Distancia, sino como un mecanismo de medir aprendizaje también en formas organizativas de la enseñanza como las clases prácticas.

Como valoración final sobre la aplicación de la técnica se puede concluir que los criterios emitidos y los resultados obtenidos mediante el criterio de usuarios permitieron corroborar que la propuesta posee alto nivel de satisfacción. 


\section{CONCLUSIONES}

1. El modelo para el diagnóstico de habilidades profesionales se orienta a determinar el grado de incertidumbre de las alternativas mediante el empleo de mapas cognitivos difusos para representar el conocimiento causal.

2. El modelo propuesto es guiado por un conjunto de principios generales y está determinado por una estructura general colaborativa que incluye el conjunto de entradas y salidas.

3. La aplicación de la técnica ladov permitió obtener el grado de satisfacción del conjunto de usuarios a los que se le aplicó el instrumento, valorando la propuesta con alta satisfacción siendo esta la máxima calificación que emite el instrumento. 


\section{REFERENCIAS BIBLIOGRÁFICAS}

Bello R, González S, Martín A, \& M, L. (2015). Modelo para el análisis estático en grafos difusos basado en indicadores compuestos de centralidad. RCCI, Vol.9(No.2), 52-65.

Castillo, J., \& Ginoris, O. (2005). Formación y desarrollo de los intereses profesionales pedagógicos en los estudiantes de primer año de la licenciatura en educación como inductores del aprendizaje autodidacto. Instituto superior pedagógico "Juan Marinello".

Francedy, D. (2011). La incorporación del e-learling en la educación superior. Congreso de Investigación y Pedagogía, pp.259.

González, M., \& Cabero, M. (2009). La evaluación por competencias: propuesta de un sistema de medida para el grado en Información y Documentación. BID, 23.

Heedy, C., \& Uribe, M. (2008). La educación a distancia: sus características y necesidad en la educación actual. Educación, Vol. XVII (33), pp. 7-27.

Kuzmina, N. (1970). Metódicas investigativas de la actividad pedagógica. Moscú, Rusia: Editorial Leningrado.

López , A., \& González, V. (2002). La técnica de ladov una aplicación para el estudio de la satisfacción de los alumnos por las clases de educación física Revista Digital - Buenos Aires.

Mar, O., Gulín, J., \& I, S. (2016). Sistema de Laboratorios a Distancia para la práctica de Control Automático. Revista Cubana de Ciencias Informáticas, Vol.10(4), 171-183.

Mar, O., I, S., \& Gulín, J. (2017). Competency assessment model for a virtual laboratory system and distance using fuzzy cognitive map. Revista Investigacion Operacional, 2(38), from http://rev-inv-ope.univ-paris1.fr/files/38217/38217-07.pdf

Sáez, M. (2012). Metodología didáctica y tecnología educativa en el desarrollo de las competencias cognitivas: aplicación en contextos universitarios. Revista de currículum y formación del profesorado, 16(3).

Samuelsen, D. A. H., \& Graven, O. H. (2016). Remote laboratories in engineering education an overview of implementation and feasability. LACCEI International MultiConference for Engineering, Education and Technology.

Santana, I., Ferre, M., Izaguirre, E., Aracil, R., \& Hernandez, L. (2013). Remote Laboratories for Education and Research Purposes in Automatic Control Systems. Industrial Informatics, IEEE Transactions on, 9(1), 547-556. doi: 10.1109/TII.2011.2182518 